\title{
Masa en el cordón espermático como primera manifestación de una vasculitis sistémica: Presentacion de un caso y revisión de la literatura
}

\author{
P. San Miguel, G.C. Fernández*, D. Pesqueira Santiago**, J.A. Ortiz-Rey, O. Durán***, \\ J.E. Dos Santos**, I. Antón \\ *Servicios de Anatomía Patológica, Radiología, **Urología y ***Medicina Interna. Centro Médico POVISA, \\ Vigo (Pontevedra).
}

Actas Urol Esp 2005; 29 (8) 777-781

\section{RESUMEN}

MASA EN EL CORDÓN ESPERMÁTICO COMO PRIMERA MANIFESTACIÓN DE UNA VASCULITIS SISTÉMICA: PRESENTACION DE UN CASO Y REVISIÓN DE LA LITERATURA.

Describimos una vasculitis granulomatosa necrotizante del cordón espermático en un varón de 35 años que presentó tres años antes un accidente cerebrovascular agudo (ACVA) de etiología no filiada. La forma de presentación clínica fue una masa no dolorosa en región testicular izquierda. El diagnóstico fue realizado en biopsia del cordón espermático. Se comentan las características clínicas, radiológicas e histológicas de este caso y se revisa la literatura.

Palabras clave: Vasculitis, testículo, infarto cerebral, diagnóstico.

\section{ABSTRACT}

SPERMATIC CORD MASS AS A MANIFESTATION OF SYSTEMIC VASCULITIS: A CASE REPORT AND REVIEW OF THE LITERATURE.

We report a case of necrotizing granulomatous vasculitis in the spermatic cord in a 35-year-old man with an antecedent of brain stroke 3 years before. The clinical manifestation was as a painless left scrotal mass. The diagnosis was established by histological examination of the spermatic cord. We discuss the physical findings, radiological features and pathological findings, reviewing the literature for previous similar cases.

Keywords: Vasculitis, testes, cerebral infarct, diagnosis.

$\mathrm{L}^{\mathrm{a}}$ a poliarteritis nodosa (PAN) es una vasculitis necrotizante de etiología no conocida que afecta a arterias y venas musculares de pequeño a mediano calibre en cualquier órgano. Los testículos están frecuentemente afectados en esta enfermedad, con una incidencia en estudios de autopsia que oscila entre el 60 al $86 \%^{1}$. Sin embargo, es excepcional que la afectación testicular sea la forma de presentación de una poliarteritis nodosa, ya que generalmente es asintomática $^{1-3}$.

Se describe un caso de vasculitis granulomatosa necrotizante del cordón espermático en un varón de 35 años, que presentó un accidente cerebro vascular agudo (ACVA) de etiología no filiada tres años antes y que clínicamente se comportó como una PAN. Describimos las características clínicas, radiológicas e histológicas y revisamos los casos de vasculitis testicular publicados en la literatura hasta la fecha.

\section{CASO CLÍNICO}

Varón de 35 años que acudió al Servicio de Urología por presentar un nódulo de $2,5 \mathrm{~cm}$. en la región paratesticular izquierda. Tres años antes, había ingresado en nuestro hospital por 
un ACVA parietal izquierdo presentando pérdida de fuerza en hemicuerpo derecho y dificultad para el habla. En la tomografía axial computerizada (TAC) de cráneo se apreció una zona hipodensa en el brazo anterior de la cápsula blanca interna izquierda, así como también otras más sutiles en la región fronto-parietal izquierda. Tras la administración del contraste no se apreciaron imágenes compatibles con aneurisma, pero se observó una tortuosidad en la arteria cerebral media derecha que podría corresponder bien a un trombo o a efecto de volumen parcial en el contexto de un vaso de morfología tortuosa. En la angio-resonancia magnética se observó que las arterias cerebrales medias eran asimétricas en cuanto a su señal, presentando la izquierda una disminución de señal en todo su trayecto, sin estenosis. El electrocardiograma no mostró alteraciones. La ecografía carotídea, arteriografía de troncos cerebrales y supraórticos y el ecocardiograma transesofágico fueron también normales. En las determinaciones analíticas sanguíneas, todos los parámetros fueron normales, incluidos los estudios realizados para llegar a un diagnóstico etiológico como: anticoagulante lúpico, homocisteína, anticuerpos anticardiolipina, $\alpha 2$ antiplasmina, anticuerpos anti-proteinasa-3, anticuerpos anti-mieloperoxidasa, proteína $\mathrm{S}$, actividad de proteína $\mathrm{C}$, RPR, anticuerpos antinucleares, anticuerpos anticitoplasma de neutrófilos (ANCA), anticuerpos anti SSA Ro y anti SSB LA. En definitiva, se trata de un paciente joven que presenta un cuadro de ACVA en el territorio de la cerebral media izquierda de etiología no filiada.

En el momento actual presenta una masa pétrea en región paratesticular izquierda. En la ecografía testicular se observa un engrosamiento del cordón espermático izquierdo. Los testículos son de tamaño y morfología normal. Se realizó un estudio de la Resonacia Magnética objetivándose en el cordón espermático izquierdo un nódulo que se comporta con alta señal en secuencias T1 y T2 (Fig. 1). Tras la administración de contraste se produce un realce periférico del mismo con una zona hipointensa en el centro que representa a una zona avascular de necrosis. Las posibilidades diagnósticas fueron dos: liposarcoma del cordón espermático o vasculitis.

Se realiza biopsia de cordón espermático y testículo. Macroscópicamente se observa una lesión nodular, sólida y de coloración blanquecina, con zona irregulares amarillentas que medía $4 \times 2,5 \times 1,5 \mathrm{~cm}$. Histológicamente se observa una vasculitis granulomatosa necrotizante que afectaba fundamentalmente a vasos de mediano tamaño y con una zona centra irregular con necrosis (Figs. 2, 3 y 4). El infiltrado inflamatorio es transmural y ocasiona una obliteración prácticamente total de la luz vascular. Periféricamente en las partes blandas adyacentes se observa marcada fibrosis, necrosis grasa e infiltrado inflamatorio agudo y crónico. Se realiza tinción con la técnica de Ziehl-Neelsen y no se observa bacilos alcohol resistentes. El diagnóstico anatomo patológico es de vasculitis granulomatosa necrotizante en zona paratesticular. En la biopsia de testículo no se identifican alteraciones significativas.

El paciente no presentó complicaciones postoperatorias y fue tratado con Deflazacort a dosis de $30 \mathrm{mg} /$ día. En el momento actual después de 16 meses de evolución el paciente no presenta ninguna sintomatología y sigue con dosis de mantenimiento (6 mg/día).

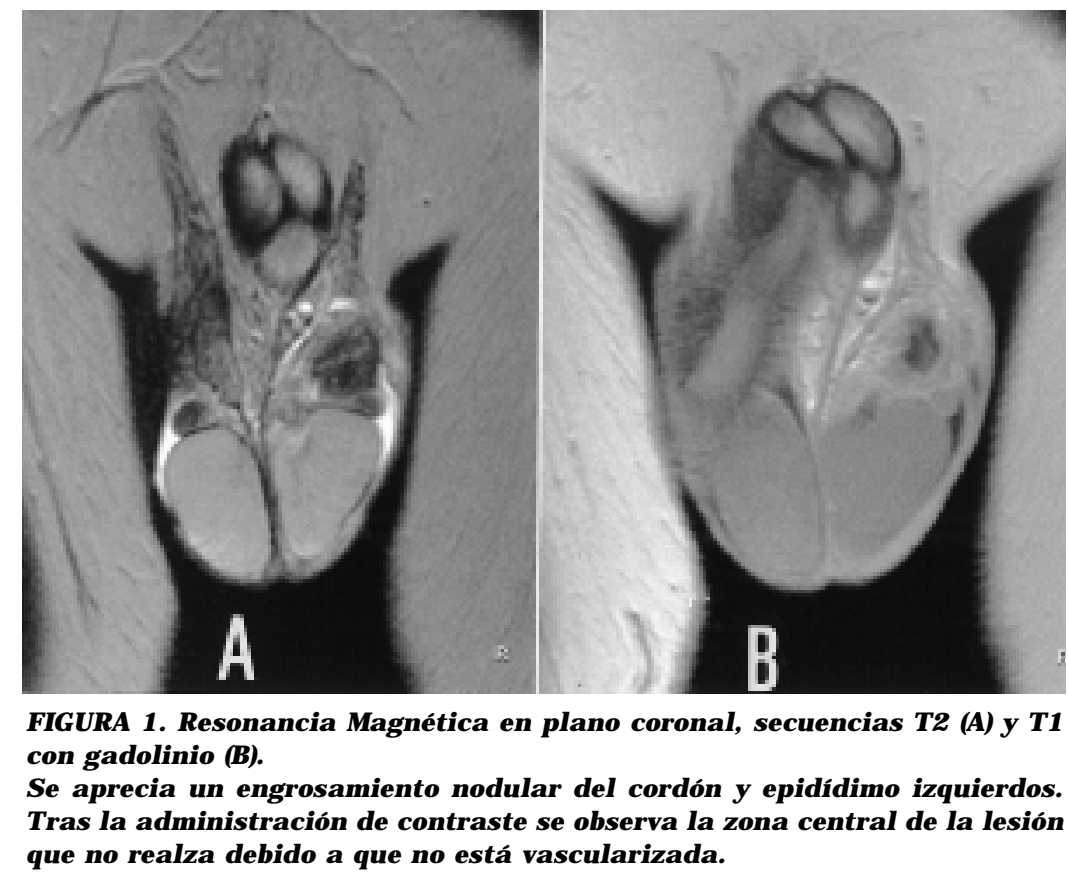




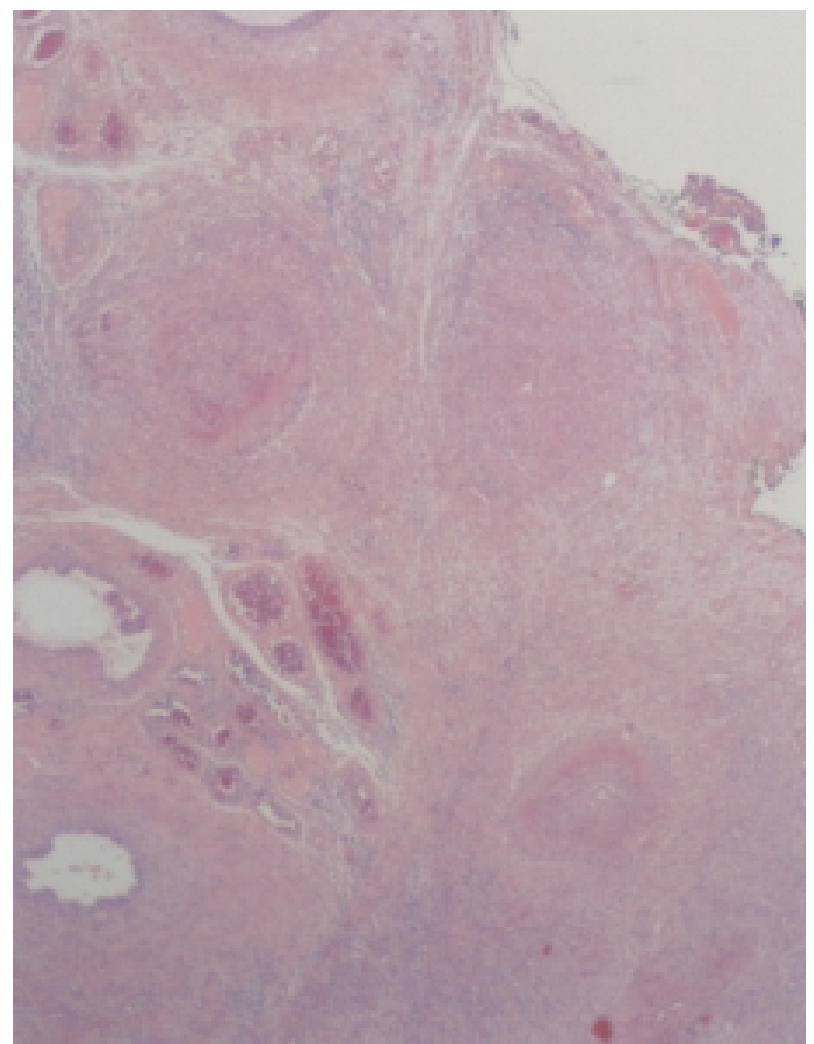

FIGURA 2. Múltiples vasos afectados por una vasculitis granulomatosa en región paratesticuar (H\&Ex50).

\section{DISCUSION}

La PAN es un proceso inflamatorio necrotizante que afecta a arterias y venas de pequeño y mediano tamaño. Es una enfermedad infrecuente con una incidencia anual del 0.7/100.000 habitantes, y ocurre principalmente en varones entre la $4^{\text {a }}$ y la $6^{\underline{a}}$ década $^{4}$. En la mayoría de las ocasiones, la afectación testicular es asintomática (sólo el 18\% de los pacientes están clínicamente afectados) ${ }^{5}$, pero hay frecuentemente repercusión histológica, que suele ser diagnosticada en autopsias $(60-86 \%)^{1}$.

La manifestación clínica de esta enfermedad es muy variada y en general predominan los síntomas constitucionales como fiebre, anemia, fatiga y pérdida de peso $^{5}$. En la PAN es frecuente la afectación testicular, pero es inusual que esté limitada al testículo ${ }^{4,5,7-15}$, o que la afectación de este órgano sea la forma de presentación de una vasculitis sistémica ${ }^{6,16}$, como es nuestro caso.

La PAN puede afectar a cualquier órgano, pero la forma de presentación como una lesión pseudotumoral parece casi exclusiva del sistema genitourinario ${ }^{17}$.

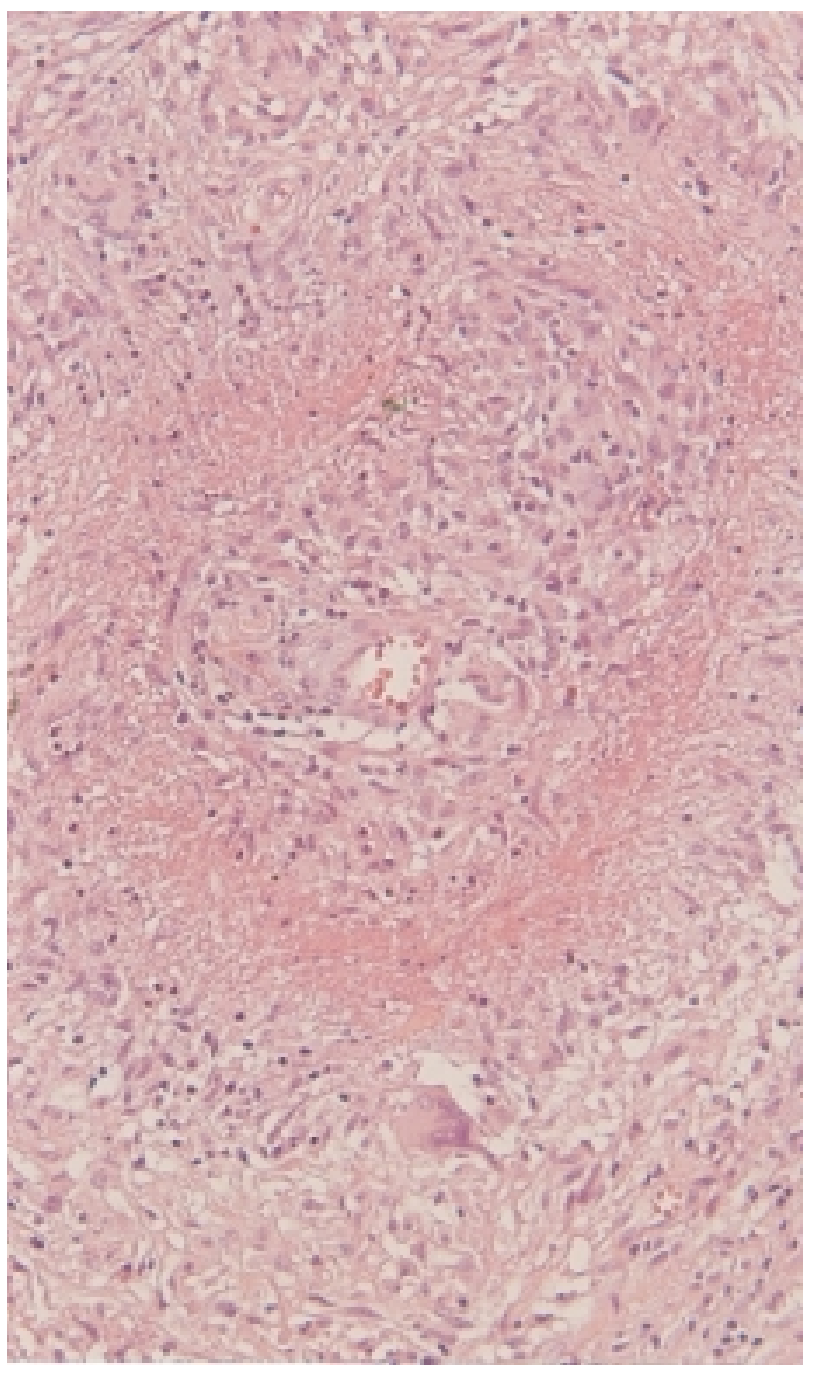

FIGURA 3. Vasculitis afectando a arterias de mediano tamaño. (H\&EX210).

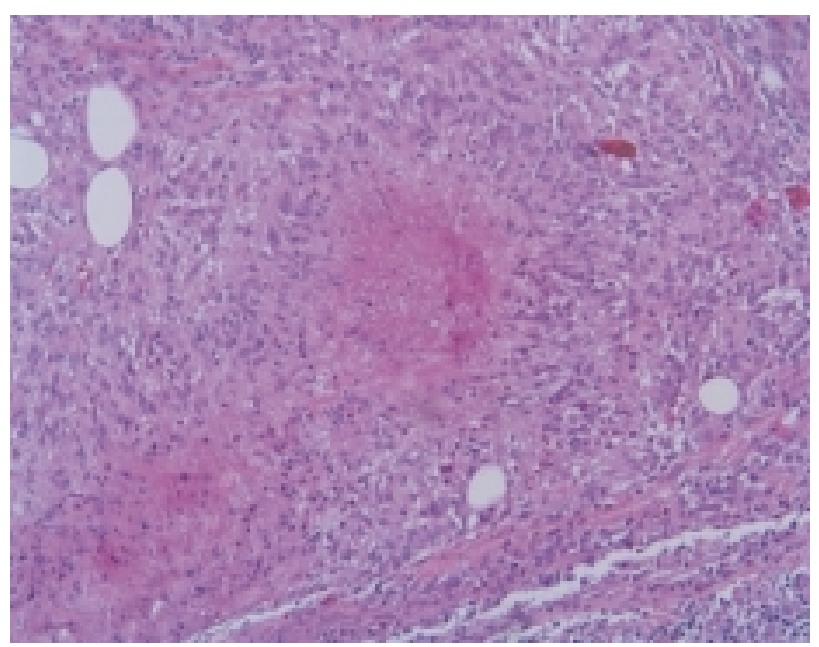

FIGURA 4. Vasculitis granulomatosa necrotizante (H\&Ex 210). 
Shurbaji y cols describieron cinco casos en los que la afectación testicular fue el primer sintoma ( 3 casos) o un signo precoz ( 2 casos) de una vasculitis sistémica $^{6}$. En tres pacientes los sintomas iniciales estaban limitados al testículo pero durante el seguimiento evolucionaron a una PAN con afectación sistémica. Se trataba de pacientes con una edad media al diagnóstico de 44 años (29-56 años); tres de ellos consultaban por fiebre y sintomas testiculares, mientras que en los otros dos casos debutaron con alteraciones neurológicas en un paciente y con proteinuria en otro. El tiempo medio transcurrido desde que apareció el primer síntoma testicular hasta la afectación sistémica fue de 31 meses ( 2 meses- 7 años). En tres casos los paciente fallecieron y la supervivencia media fue de 56 meses (2-137 meses). En nuestro caso, el primer síntoma fue también neurológico, pero el diagnóstico de vasculitis fue realizado tres años después cuando el paciente presentó una masa paratesticular que histológicamente fue tipificada como vasculitis granulomatosa necrotizante. Actualmente después de 14 meses de seguimiento el paciente está sano y asintomático.

No obstante, a pesar de que el comportamiento clínico es el de una vasculitis tipo PAN; histológicamente es una vasculitis granulomatosa necrotizante que es más típica de una granulomatosis de Wegener (GW). La GW es una vasculitis necrotizante que afecta fundamentalmente al aparato respiratorio superior, inferior y riñón. Aunque potencialmente puede afectar a cualquier órgano, la afectación urogenital es rara si exceptuamos el riñón. Davenport y $\operatorname{cols}^{19}$ han descrito 8 casos de GW con afectación urogenital y sólo en un caso hubo afectación testicular con ANCA positivos. Según la literatura revisada, hay dos casos más de GW con afectación testicular y epididimaria $^{10,20}$. En un caso, el paciente tenia también afectación pulmonar y ANCA positivos ${ }^{20}$ y en el otro caso había afectación limitada al epidídimo y ANCA negativos ${ }^{19}$. En nuestro caso, las características clínicas de la lesión, la inusual afectación del testículo en esta enfermedad y lo que es más importante, la negatividad para los ANCA descartó clínicamente el diagnóstico de GW. En resumen, el diagnostico final fue de una PAN, aunque la histología es mas característica de una vasculitis granulomatosa de Wegeger.
Este caso nos confirma que a pesar de los grandes avances en la etiopatogenia, diagnóstico de laboratorio y de imagen, la clasificación de las vasculitis todavía no esta resuelta y que a pesar de que el diagnóstico de vasculitis queda casi siempre bien establecido, como es nuestro caso, hay dificultad para su adscripción en un tipo concreto $^{21}$.

Nuestro caso sugiere que una masa testicular o paratesticular, sobre todo en pacientes jóvenes, puede ser la forma de presentación de una vasculitis sistémica. Según la literatura revisa$\mathrm{da}^{4,7,8,10-8,12,14}$ no es infrecuente que las vasculitis se manifiesten como masas simuladoras de tumores y por lo tanto se realizan cirugías agresivas con finalidad curativa ${ }^{4,6,7,8,9,12,14, \text { En }}$ nuestro caso, aunque la forma de presentación fue una masa en región paratesticular dos fueron los diagnósticos posibles por métodos de imagen: liposarcoma o vasculitis. El primero se sugirió por ser hiperintenso en señal T1, que es el comportamiento usual de la grasa y la segunda opción diagnóstica se sospechó por los antecedentes clínicos y la edad del paciente. Por este motivo se realizó el estudio mediante biopsia intraoperatoria y se realizó una cirugía conservadora.

\section{CONCLUSIÓN}

En resumen, describimos una forma de presentación inusual de vasculitis sistémica y comentamos la necesidad de hacer diagnóstico diferencial entre proceso neoplásico y vasculitis en las masas genitourinarias antes de realizar una cirugía agresiva, sobre todo si son pacientes jóvenes.

\section{REFERENCIAS}

1. Dahl EV, Baggenstoss AH, DeWeerd JH. Testicular lesions of periarteritis nodosa, with special reference to diagnosis. Am J Med 1960;28:222-228.

2. Valleteau de Moulliac M, Molle D. Circumstances of unusual discovery of periarteritis nodosa. Nouv. Presse Med., 1977; 6:4069.

3. Wright LF, Bicknell SL. Systemic necrotizing vasculitis presenting as epididymitis. J Urol 1986;136 (5):1094.

4. Eilber KS, Freedland SJ, Rajfer J. Polyarteritis nodosa presenting as hematuria and testicular mass. J Urol 2001;166: 624 .

5. Tanuma Y, Oda T, Yokoo A, Ito S, Taeuchi K. Recurrent polyarteritis nodosa limited to the testis. J Urol 2003; 170: 1953. 
6. Shurbaji MS, Epstein JI. Testicular vasculitis. Implications for systemic disease. Hum Pathol 1988;19:186-189.

7. Huisman TK, Collins WT, Voulgarakis GR. Poyarteritis nodosa masquerading as a primary testicular neoplasm:a case report and review of the literature. J Urol 1990;144: 1236-1238.

8. Mowad JJ, Badwin BJ, Young JD. Periarteritis nodosa presenting as a mass in the testis. J Urol 1971;105:109111 .

9. Persellin ST, Menke DM. Isolated polyarteritis nodosa of the male reproductive system. J Rheumatol 1992;19:985988.

10. Rowling SE, Shapiro ML, Llieberman AP, Coleman BG. Intratesticular vasculitis simulating a testicular neoplasm. J Ultrasound Med 1996;15:161-163.

11. Inoue $Y$, Yamashita $M$, Shuin $T$. Localized polyarteritis nodosa presenting as epididymal mass: a case report. Hinyokika Kiyo 1997;43:895-897.

12. Warfield AT, Lee SJ, Phillips SM, Pall AA. Isolated testicular vasculitis mimicking a testicular neoplasm. J Clin Pathol 1994;47:1121-1123.

13. Mukamel E, Abarbanel J, Savion M, Konichezky M, Yachia D, Auslaender L. Testicular mass as a presenting symptom of isolated polyarteritis nodosa. Am J Clin Pathol 1995; 103:215-217.

14. Kessel A, Toubi E, Golan TD, Toubi A, Mogilner JG, Jaffe M. Isolated epididymal vasculitis. Isr Med Assoc J 2001; 3:65-66.
15. Kishino T, Fujimoto K, Hayashi Y, Momose H, Otani T, Ozono S, Hirao Y, Shiiki H, Dohi K, Maruyama H. A case of polyarteritis nodosa preesenting as a mass in scrotum. Hinyokika kiyo 2001;47:211-213.

16. Dotan ZA, Laufer M, Heldenberg E, Langevitz P, Fridman $\mathrm{E}$, Duvdevani M, Ramon J. Isolated testicular polyarteritis nodosa mimicking testicular neoplasm-long-term followup. Urology 2003;62:352.

17. Kariv R, Sidi Y, Gur H. Systemic vasculitis presenting as a tumorlike lesion. Medicine 2000;79:349-359.

18. Davenport A, Downey SE, Goel S, Maciver AG. Wegener's granulomatosis involving the urogenital tract. Brit J Urol 1996;78:354-357.

19. Al-Arfaj A. Limited Wegener's granulomatosis of the epididymis. Int $\mathrm{J}$ Urol 2001;8:333-335

20. Agraharkar M, Gokhale S, Gupta R. Wegener's granulomatosis diagnosed by testicular biopsy. Int Urol and Nephrol 2002;34:559-564.

21. Barbado Hernández FJ, Gómez Cerezo J, Rios Blanco JJ, Vázquez Rodríguez JJ. Más dificultades en la clasificación de las vasculitis. Med Clin (Barc) 2002;1:394-400.

Dra. P. San Miguel Fraile.

Servicio de Anatomía Patológica. Hospital POVISA.

C/ Salamanca no 5, 36211 Vigo (Pontevedra)

e-mail:psanmiguel@povisa.es

(Trabajo recibido el 9 diciembre 2004) 